\title{
Improving WiMAX Capacity Of A Cell By Applying Relay Stations And Adaptive Antenna Systems.
}

\author{
Dr. A. I. A. Jabbar \\ Assistant Professor \\ Department of Electrical \\ Engineering \\ Mosul university
}

\author{
Firas S. Al-Sharbaty \\ M.Sc. Student \\ Department of Electrical \\ Engineering \\ Mosul university
}

\begin{abstract}
Worldwide interoperability for Microwave $\underline{A}$ ccess (WiMAX) is a broadband wireless technology based on IEEE 802.16 standard . A WiMAX cell which is to be considered in this study applies multicast mode of operation, the main limitation of maximizing the capacity of the system is the signal to noise ratio (subscribers near the base station apply 64 QAM and $3 / 4$ Reed Solomon code, while those which are near the border of the cell applies PSK and $1 / 2$ Reed Solomon code to compensate for the $S / N$ effect). Results indicate that better capacity is achieved by introducing Relay Stations (RSs) and Adaptive Antenna System (AAS) to the cell with TDD mode of operation, other factors like MAC and PHY layers overheads are studied and taken into account in the calculation of the system capacity .
\end{abstract}

Keywords : WiMAX, Overhead, Capacity, AAS , Relay Stations .

$$
\text { تحسين سعة خلية الو ابي ماكس بأستخدام المرحلات و أنظمة الهو ائيات المحدثة }
$$

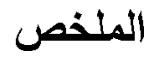

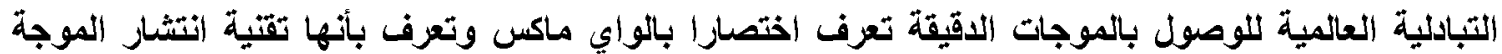

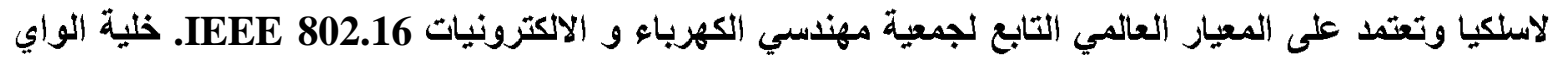

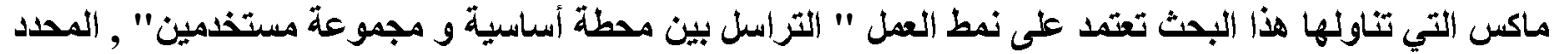

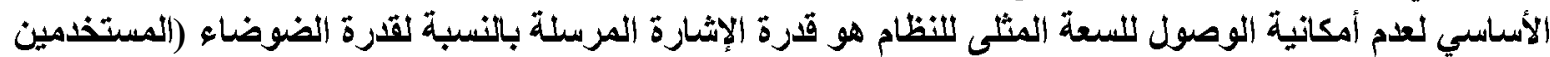

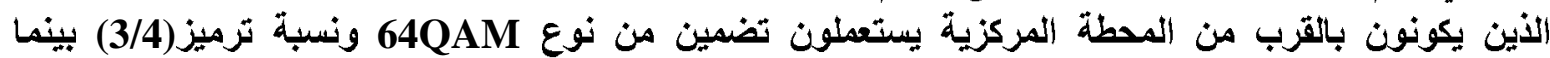



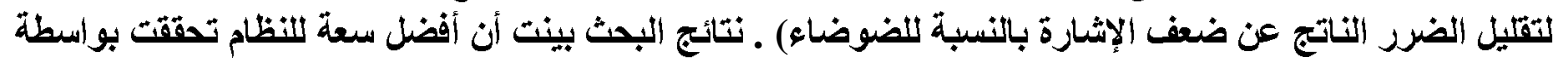

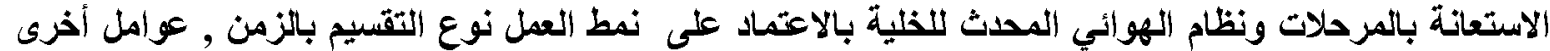






\section{1-Introduction}

WiMAX systems are based on the IEEE 802.16d-2004 for fixed services and IEEE802.16e-2005 standards for mobile services, they define a physical (PHY) and a medium access control (MAC) layers for broadband wireless access systems operating at frequencies below $11 \mathrm{GHz}$. Relay stations are introduced to increase the range and system capacity, they allow multihop communication, which occurs when data travel from the source to the destination node via intermediate nodes. Hence, range of the network can be significantly extended without installment of other costly BSs. The traffic always leads from or to BS, thus direct communication between Subscriber Stations (SSs) is not considered.

Adaptive antenna system (AAS) is an optional feature, it enhances the coverage and capacity as well as spectral efficiency. It has the advantage of applying only one antenna at subscriber station (SS) side, and as a consequence it simplifying the SS implementation procedure .

This paper deals with fixed WiMAX system which is based on IEEE 802.16d-2004 standard, it analyzes the overhead during system configuration especially those related to the layers (PHY and MAC layers), also the capacity and the throughput of the system are estimated with and without Adaptive Antenna System .

\section{2-Theory}

\section{2-1-PHY Layer}

The overhead related to the physical layer should be determined to evaluate the capacity of WiMAX system. According to [1], the channel bandwidth may vary from $1.75 \mathrm{MHz}$ to $28 \mathrm{MHz}$. The bigger channel size can provide more radio resources and thus capacity of the system is larger .

To prevent inter symbol interference (ISI) caused by delay spread, part of the OFDM symbol is appended at the beginning of the symbol. The repeated symbol fraction is called cyclic prefix (CP) and its size depends on type of environment. From bandwidth size and CP values, symbol time duration ( time of one symbol) can be derived .

In case of OFDM modulation used in 802.16 standards, the bandwidth is divided into 256 subcarriers. When pilot and null subcarriers are subtracted, the remaining 192 subcarriers can be used to carry user's data. The capacity of each subcarrier depends on the order of used modulation. WiMAX standard supports BPSK (1 bit per subcarrier), QPSK (2 bits per subcarrier), 16QAM (4 bits per subcarrier) and finally 64 QAM (6 bits per subcarrier)[2].

Because of errors introduced by imperfection of the channel, Reed Solomon is used, it provides redundant bits which must be carried with useful information for the purpose of error detection and correction at the receiver. The ratio of information to information plus redundant bits is called coding rate and may vary from $1 / 2$ to $3 / 4$. WiMAX parameters are demonstrated in Table (1). The parameters values shows that overhead introduced by the PHY layer is considerable [2] . 


\begin{tabular}{|c|c|}
\hline PARAMETERS & VALUE \\
\hline BANDWIDTH BW $(\mathrm{MHz})$ & 20 \\
\hline symbol useful time $(\mu \mathrm{s})$ & 11.64 \\
\hline CP time $(\mu \mathrm{s})$ & 2.91 \\
\hline overall symbol time $(\mu \mathrm{s})$ & 14.55 \\
\hline OFDM Subcarriers & 256 \\
\hline Data Subcarriers & 192 \\
\hline $\begin{array}{c}\text { At coding rate1/2, PHY } \\
\text { OVERHEAD }(\%)\end{array}$ & 71.88 \\
\hline $\begin{array}{c}\text { At coding rate3/4, PHY } \\
\text { OVERHEAD }(\%)\end{array}$ & 57.81 \\
\hline DUPLEX TYPE & TDD \\
\hline Access & FIXED \\
\hline
\end{tabular}

\begin{tabular}{|c|c|}
\hline PARAMETERS & $\underline{\text { VALUE }}$ \\
\hline Number of BS & 1 \\
\hline Number of RS & 2 \\
\hline Number of SSs & 50 \\
\hline BS range radius (Km) & 3 \\
\hline BS height ( m) & 30 \\
\hline SSs height ( m ) & 2 \\
\hline BS transmit power pt (dBm) & 30 \\
\hline RS transmit power p (dBm) & 26 \\
\hline Frame duration (ms) & 20 \\
\hline frequency band (GHz) & 5 \\
\hline $\begin{array}{c}\text { Two elements antenna array } \\
\text { gain ( dBi) }\end{array}$ & 18 \\
\hline \hline
\end{tabular}

Table (1) shows the value of WiMAX (802.16d-2004) parameters

\section{2-2-MAC Layer}

TDD (for licensed and licensed-exempt band) and FDD (for licensed band only) duplexing techniques are supported by the MAC layer protocol in IEEE 802.16 standard. According to TDD duplex method, every frame is divided into DL and UL sub frames . Figure (1) demonstrates TDD frame structure that divided into UL and DL sub frames [3] .

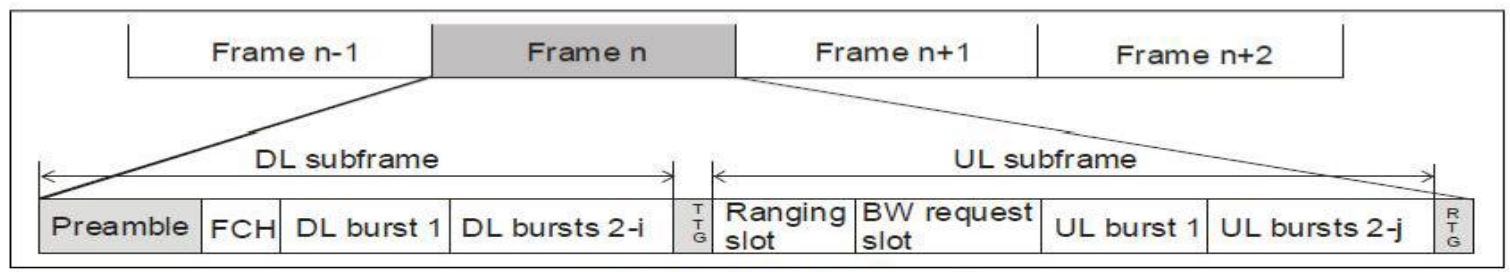

Figure (1)802.16d-2004 MAC

Every TDD frame has to start with long preamble through which SSs may synchronize to the network. After DL long preamble, the Frame Control Header (FCH) follows. It contains DL_Frame_Prefix (DLFP) which details up to 4 DL bursts (location and used burst profile type) [4].

The first DL burst includes MAC management broadcast message, e.g. UL-MAP, DL-MAP, UCD (Uplink Channel Descriptor), DCD (Downlink Channel Descriptor) . In case that DL sub-frame consists of less than five bursts, DL-MAP message can be excluded but must be sent periodically to maintain synchronization [2][4].

The DL-MAP MAC message defines how to access DL channel. It is composed of 8 bytes long header and certain number of information elements (IEs) that are 4 bytes long. There is one IE for each DL burst in every DL subframe. The DL-MAP IEs reference a specific MAC connection (CID) and a burst profile code (DIUC) so that subscriber station can know whether burst contains traffic destined for it or not. The IE further indicates if bursts start with short preamble for purpose of advanced synchronization and channel estimation[2]. 
After the DL-MAP (if present), the UL-MAP message is followed . It characterizes and schedules UL subframe. The uplink map contains 7 bytes of header information followed by 6 bytes long IEs [2].

TTG ( transmit /receive transition) gap must be inserted between downlink and uplink subframes to allow BS to switch from transmit to receive mode and SSs to switch from receive to transmit mode. After the uplink subframe ends, the RTG (receive/transmit transition) gap is inserted.

On the other hand, DL and UL bursts must consist of integer number of OFDM symbols. To accomplish this, every unused byte in symbol must be padded by the bytes 0xFF [2][4] .

\section{2-3-The Performance of Relay Station (RS)}

WiMAX like other wireless systems suffers from radio propagation characteristics. The achievable signal-to-noise ratio (SNR) decreases with an increasing link distance. This results in low SNR at the cell border. Shadowing, which leads to non line of-sight (NLOS) communication, further reduces the signal quality. The introduction of relay stations may significantly enhance the link quality leading to throughput enhancements and coverage extensions. Thus, relays allow providing broadband access to subscribers in remote places. Furthermore, a network can be flexibly adapted to changing users' behavior or environmental conditions. Two concepts to integrate multihop communication into IEEE 802.16 standard are possible. The first one follows a centralized approach, where the BS has full control over the relay-enhanced cell. The second concept follows a semi-distributed approach, where RS coordinates the performance of the associated SSs. In the second case, the MAC protocol complexity of RS is comparable to BS. This paper deals with centrally controlled RS (first concept)[3][5], see Figure(2) .

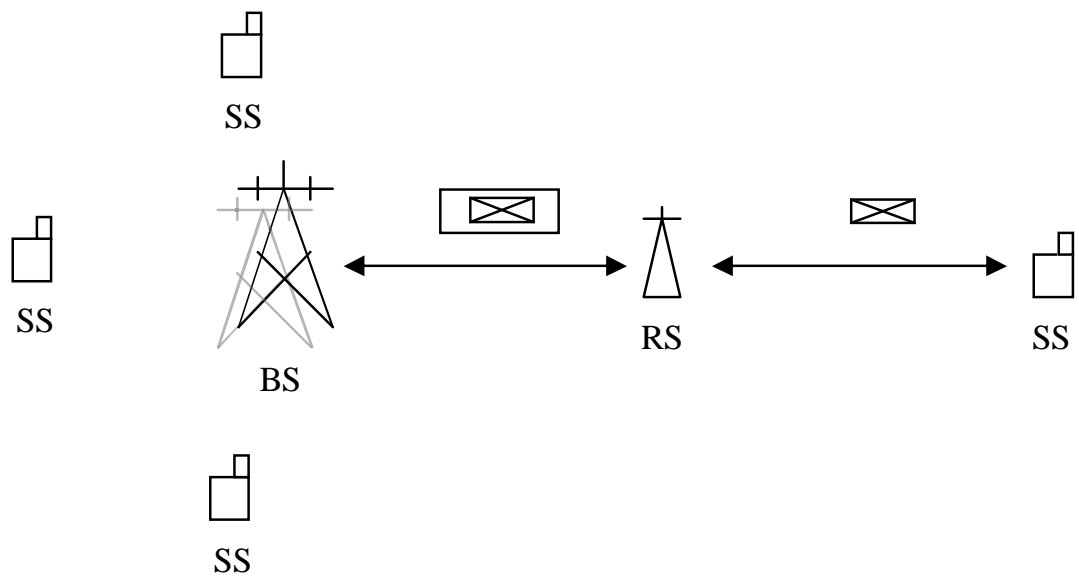

Figure (2) WiMAX network with Relay Station .

\section{2-3-1-MAC Frame of Centrally Controlled RS}

Figure (3) shows MAC frame structure, when BS cell is enhanced by centrally controlled RS. The beginning of the frame is similar to that in IEEE 802.16 standard. This means that DL frame starts with long preamble followed by FCH. Since the RSs are centrally controlled by the BS, the DL and UL-MAP's broadcasted by the BS have to schedule transmission on the first and also on the second hop. Consequently, in comparison with previous case, the maps have to be larger. After broadcast information is received, the RS filters it and forwards only information relevant to its associated stations. Between BS and RS broadcasts, the gap has to be inserted because of round 
Jabbar: Improving WiMAX Capacity Of A Cell By Applying Relay Stations

trip delay and to allow RS switch from receive to transmit mode together with filtering time [3].

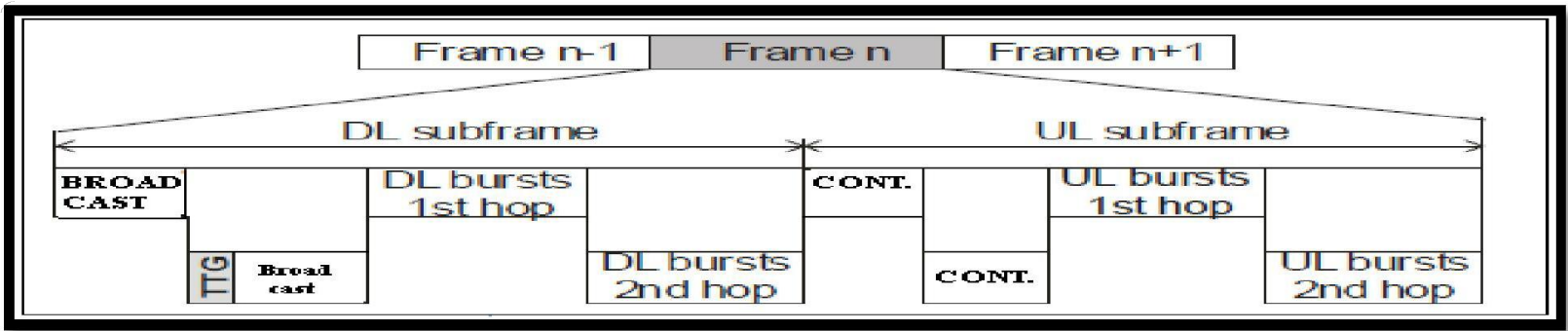

Figure (3) MAC frame centrally RS.

\section{2-4-Adaptive Antenna Systems}

A smart antenna system combines an antenna array with digital signal processing capability to transmit and receive in a predefined or adaptive, spatially sensitive manner. This enables such a system to change the directionality of its radiation patterns in response to a particular signal environment. This result dramatically increases the performance characteristics of a wireless system.

A typical smart antenna array of a $120^{\circ}$ segment with one, 2, 4 or 8 antenna elements increases the gain and narrowing the azimuth beamwidth with the increase in antenna elements is clearly illustrated in Figure (4), Table (2) reveals the effect of increasing the number of antenna elements on the gain and AZ [6] .

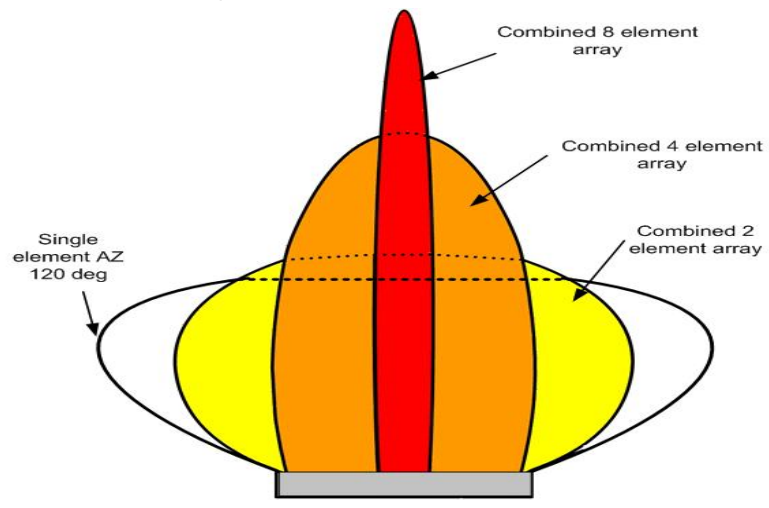

Figure (4) Single, 2, 4 and 8 Element Performance Illustration[6]

\begin{tabular}{|c|c|c|}
\hline Antenna elements & $\underline{\text { Gain }}$ & $\underline{\underline{\boldsymbol{Z Z}}}$ \\
\hline One element & $15 \mathrm{dBi}$ & $120^{\circ}$ \\
\hline 2 elements & $18 \mathrm{dBi}$ & $60^{\circ}$ \\
\hline 4 elements & $21 \mathrm{dBi}$ & $30^{\circ}$ \\
\hline 8 elements & $24 \mathrm{dBi}$ & $15^{\circ}$ \\
\hline
\end{tabular}

Table (2)shows the values of the maximum gain and AZ as

a function of the number of antenna's elements.

\section{2-4-2-MAC Frame Support For AAS (Adaptive Antenna System).}

IEEE 802.16 standard devotes a downlink frame by what is called the AAS zone which is dedicated to AAS traffic, as illustrated in Figure (5). This allows a mixture of 
AAS and non-AAS users to be supported by the same Base Station (BS). Which dynamically allocates capacity to AAS and non-AAS traffic. SSs that have no AAS capability simply ignore the traffic in an AAS zone [7] .



Figure (5) MAC frame supports AAS.

\section{3- Simulation Model Description and Analysis}

\section{3-1-PHY Overhead Calculation}

Cyclic prefix, coding rate and 64 subcarriers (pilot and null subcarriers) from 256 subcarriers represent the PHY layer overhead .The details are shown in Table (3).

\begin{tabular}{|c|c|}
\hline Parameter & $\underline{\text { Value }}$ \\
\hline Bandwidth( MHz) & 20 \\
\hline OFDM subcarriers & 256 \\
\hline Data subcarriers & 192 \\
\hline null and pilot subcarriers & 64 \\
\hline Symbol useful time $(\mu \mathrm{s})$ & 11.64 \\
\hline cyclic prefix time $(\mu \mathrm{s})$ & 2.91 \\
\hline Symbol time $(\mu \mathrm{s})$ & 14.55 \\
\hline Coding rate & $1 / 2 \quad 3 / 4$ \\
\hline PHY overhead $(\%)$ & $71.88 \quad 57.81$ \\
\hline
\end{tabular}

Table (3) demonstrate the parameters of PHY layer overhead.

\section{3-2-MAC Overhead Calculations}

\section{3-2-1- Calculation Of OFDM Symbols without RS and AAS}

The overall number of OFDM symbols in one MAC frame depend on the duration of MAC frame and length of OFDM symbol, it can be calculated by the following formula (see Figure 6) [8][10] :

$\Phi=\mathrm{T}-\sum_{i=1}^{5} L i$

Where :

$\Phi=$ Number of OFDM symbols that used for data transmission without AAS and RSs.

$\mathrm{T}=$ The overall number of OFDM symbols in one MAC frame.

$\mathrm{L} 1=$ represents $\mathrm{BS}$ broadcast field including long preamble frame control header and DL/UL MAPs. Since the broadcast field of the frame is transmitted by the most robust modulation type and coding rate, only 96 bits/OFDM can be carried (see Table(5)). The length of the maps and the quantity of OFDM symbols depends on the number of IEs in MAPs.

L2 $=$ TTG = A gap between DL and UL subframes depends mainly on the round trip delay.

$\mathrm{L} 3$ = Contention slots composed from initial ranging slot and BW request slots .

L4 = Short preamble appended before every UL burst . 
L5 = A gap between the UL and DL subframes (same as L2).

It is worth mentioning that the overhead introduced by other MAC messages as well as by MAC PDU (packet data unit) headers is neglected since the impact on overall throughput is meaningless .



Figure (6) Details of OFDM symbols used for data(without RS and AAS).

The different values of the parameters of equation (1) are given in table (4) .

\begin{tabular}{|c|c|}
\hline PARAMETERS & VALUE(SYMBOLS) \\
\hline PREAMBLE OF L1 & 2 \\
\hline FCH OF L1 & 1 \\
\hline L2 & 1 \\
\hline CONTENTOIN SLOT OF L3 & 7 \\
\hline FOR ALL 5 USERS,BW REQUEST \\
OF L3 & 2 \\
\hline L4 & 1 \\
\hline L5 & 1 \\
\hline
\end{tabular}

Table(4) parameters of equation(1).

The size (in symbols) of DL and UL MAP's are given by [9] :

$\mathrm{DLm}=(64+32 * \mathrm{~S}) / 96$

$\mathrm{ULm}=(56+48 * \mathrm{~S}) / 96$

Given that :

DLm $=$ Downlink map .

ULm = Uplink map .

$\mathrm{S}=$ represents the total number of active SSs which are served in one MAC frame .

\section{3-2-2-Calculation Of OFDM Symbols with RSs and AAS}

Figure (7) demonstrates the overhead caused by RS and AAS, the number of OFDM symbols used for data can be calculated by the following formula[8][10]:

$\Psi=\mathrm{T}-\mathrm{L} 1-\sum_{i=3}^{7} L i$

Where:

$\Psi=$ represents the OFDM symbols that used for transmission with RSs and AAS.

L1 , L3 , L4 ,L5 the same as in equation (1).

L2 is discarded because BS has enough time to switch from Tx to Rs during the second hop .

L6 $=$ the overhead caused by RS , see Table (6) .

$\mathrm{L} 7=$ the overhead caused by AAS . 


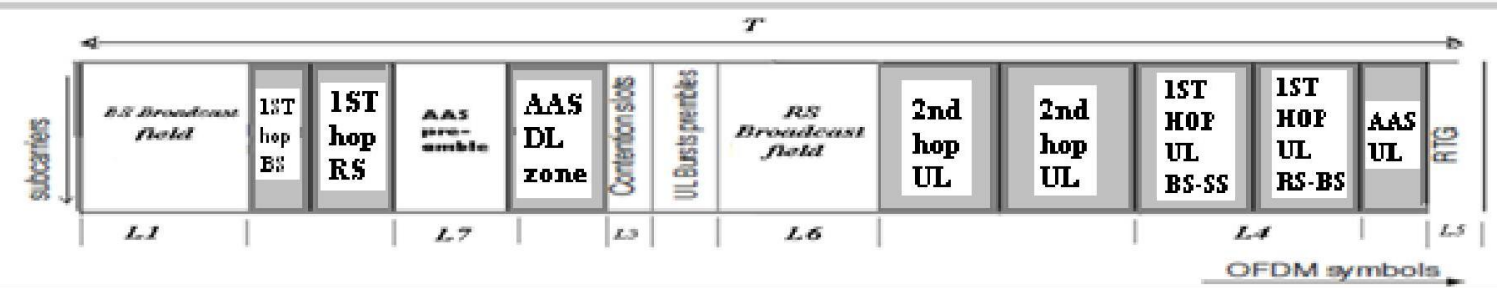

Figure (7) Details of OFDM symbol used for data(with RS and AAS).

L7 = 13 symbols

L7 contains long preamble, AAS_DLFP and optional diversity zone (send for two RSs) as well as network entry region with optional short preamble at UL subframe .

\section{3-3-Throughput And Capacity Calculation}

The useful symbols can be calculated by the subtraction of PHY and MAC layer overheads from the overall length of frame, they are used for data transmission. It is obvious that the overall system capacity is related to the number of useful symbols. System capacity depends on the modulation type and coding rate that can be applied to a single SSs (according to the received SNR). The WiMAX capacity to requested capacity can be estimated by the following equations[8][10] :

$$
\begin{aligned}
& \mathrm{Sc} / \operatorname{Rc} \Phi=\frac{\Phi}{R E Q(\Phi)} \\
& \mathrm{Sc} / \operatorname{Rc} \Psi=\frac{\Psi}{R E Q(\Psi)}
\end{aligned}
$$

Where :

$\mathrm{Sc} / \mathrm{Rc} \Phi=$ the WiMAX system capacity to requested capacity (without RS).

$\mathrm{Sc} / \mathrm{Rc} \Psi=$ the system capacity to requested capacity with $\mathrm{RS}$.

$\operatorname{REQ}(\Phi)$ and $\operatorname{REQ}(\Psi)$ represent how many OFDM symbols that are needed to transfer a given nominal bit rates for all active users.

The REQ $(\Phi)$ can be estimated from following formula[8][10]:

$\operatorname{REQ}(\Phi)=\mathrm{BR} * \operatorname{Lof} *\left(\sum_{i=1}^{7} \frac{S S(B S)}{b p s i}\right)$

Where :

$\mathrm{BR}=$ the nominal bit rate in bits per second .

Lof $=$ frame duration in second .

SS(BS) represents the number of SSs that use individual modulation type and coding rate and finally bpsi express how many bits can be allocated to one OFDM symbol .

$\operatorname{REQ}(\Psi)$ is computed by $[8][10]$ :

$\mathrm{REQ}(\Psi)=\mathrm{BR} * \operatorname{Lof} *\left[\left(\sum_{i=1}^{7} \frac{S S(B S)}{b p s i}\right)+\left(\sum_{i=1}^{7} \frac{S S(R S)}{b p s i}\right)+\left(\frac{1}{b p s} \sum_{i=1}^{7} S S B R\right)\right]$ 
Where SS(Rs) corresponds to the number of SSs on the 2nd hop (between the RS and SS). The last term in the round bracket $(S S B R)$ represents the transmission between the BS and RS and so bps express the quantity of bits that can be allocated to one OFDM symbol (dependent on the Rs-Bs link quality)[8].

The throughput per user can be derived for the cases without and with AAS and RS From equations (5) and (6) respectively as follow :

$\mathrm{BR}=\frac{\operatorname{REQ}(\Phi)}{\operatorname{Lof} *\left(\sum_{i=1}^{7} \frac{S S(B S)}{b p s i}\right)}$

$\mathrm{BR}=\frac{\operatorname{REQ}(\Psi)}{\operatorname{Lof} *\left[\left(\sum_{i=1}^{7} \frac{S S(B S)}{b p s i}\right)+\left(\sum_{i=1}^{7} \frac{S S(R s)}{b p s i}\right)+\left(\frac{1}{b p s} \sum_{i=1}^{7} S S B R\right)\right]}$

\section{4-Model Assumptions}

The WiMAX system performance is based on the following assumptions :

1- WiMAX cell consists of one BS and up to $50 \mathrm{SSs}$ (without AAS and RS).

2- The positions of SSs are chosen randomly, see Figure (8).

3 - The modulation type and coding rate depend on the geographical distribution of subscribers around the BS. see Table(5).

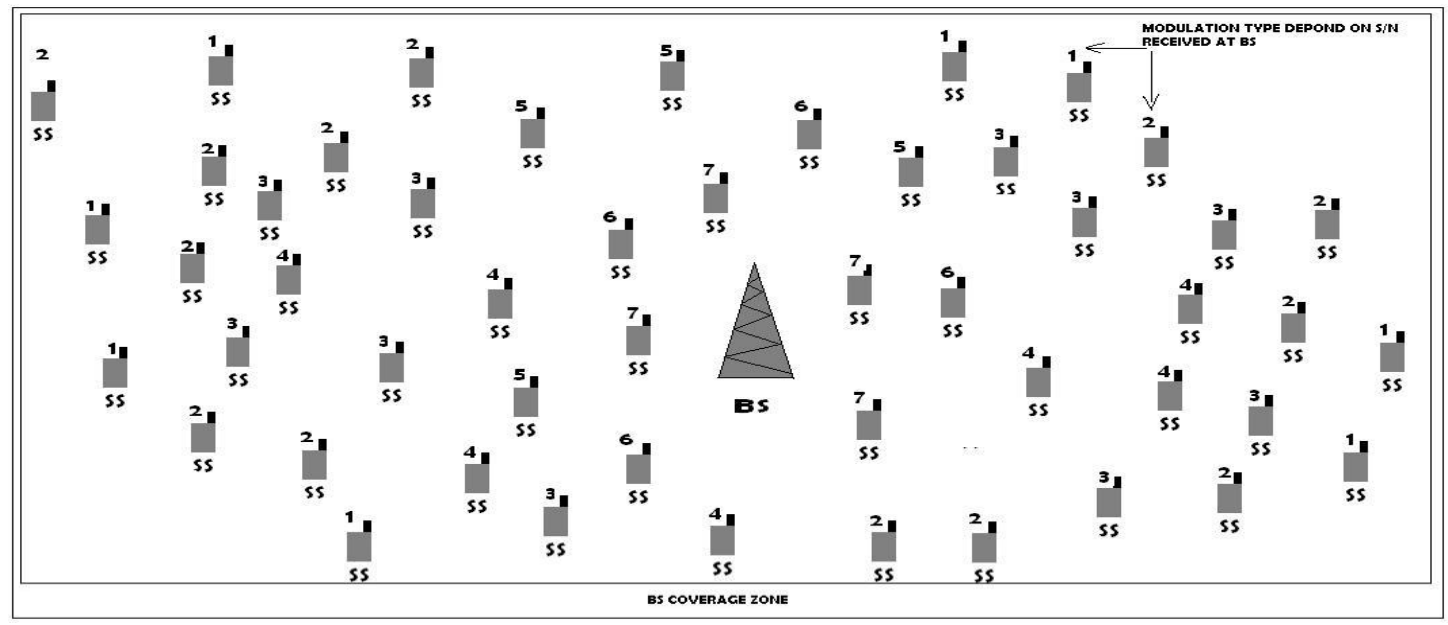

Figure (8) demonstrates the positions of SSs in first phase of simulation.

\begin{tabular}{|c|c|c|c|c|c|c|}
\hline & $\begin{array}{c}\text { Modulation } \\
\text { type }\end{array}$ & $\begin{array}{c}\text { Coding } \\
\text { rate }\end{array}$ & $\begin{array}{c}\text { S/N } \\
\text { received(dB) }\end{array}$ & $\begin{array}{c}\text { No. of SSs without } \\
\text { AAS \& RS }\end{array}$ & $\begin{array}{c}\text { No. of SSs with } \\
\text { AAS \& RS }\end{array}$ & Bits/Symbol \\
\hline 1 & BPSK & $1 / 2$ & 3 & 8 & 4 & 96 \\
\hline 2 & QPSK & $1 / 2$ & 6 & 12 & 6 & 192 \\
\hline 3 & QPSK & $3 / 4$ & 8.5 & 10 & 6 & 288 \\
\hline 4 & 16 QAM & $1 / 2$ & 11.5 & 8 & 7 & 384 \\
\hline 5 & 16 QAM & $3 / 4$ & 15 & 4 & 8 & 576 \\
\hline 6 & 64 QAM & $2 / 3$ & 19 & 4 & 10 & 768 \\
\hline 7 & 64 QAM & $3 / 4$ & 21 & 4 & 9 & 864 \\
\hline
\end{tabular}

Table (5) Usage of burst type dependence on SNR. 
4- In the second part of simulation, two SSs are converted to RSs and linked to BS using AAS (Figure (9) ).

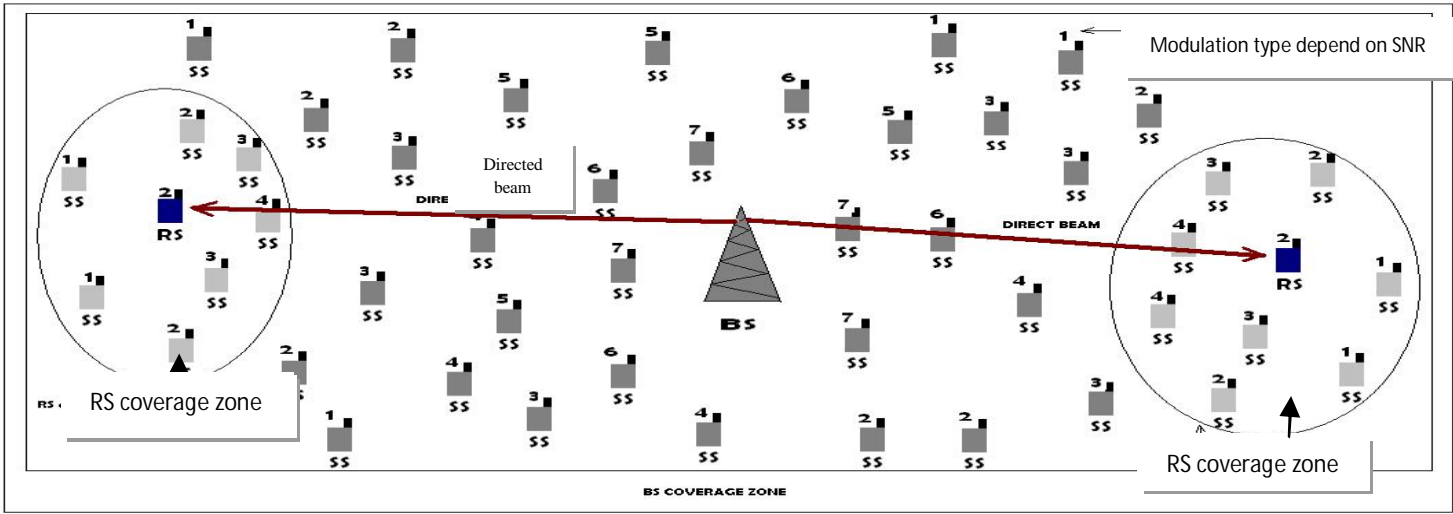

Figure (9) shows the model in $2^{\text {nd }}$ phase of simulation .

5- Nominal bit rates $=(1,2,3,4) \mathrm{Mbps}$.

6- Length of frame (Lof) $=20 \mathrm{~m} \mathrm{sec}$.

7- The UL bursts number is assumed to be equal to the eighth of user's number .

8- The values of overhead caused by RS (L6) are demonstrated in Table (6) [3] .

\begin{tabular}{|c|c|}
\hline$\underline{\text { L6 (OVERHEAD OF RSs })}$ & $\underline{\text { NUMBER OF SSS }}$ \\
\hline 35 & 5 \\
\hline 40 & 10 \\
\hline 40 & 15 \\
\hline 40 & 20 \\
\hline 43 & 25 \\
\hline 45 & 30 \\
\hline 48 & 35 \\
\hline 49 & 40 \\
\hline 51 & 45 \\
\hline 53 & 50 \\
\hline
\end{tabular}

Table (6) Overhead of L6 with number of SSs .

\section{5-Simulation Results}

Figure (10) demonstrates the percentage of MAC layer overhead (without RSs \& AAS) as a function of the number of users .

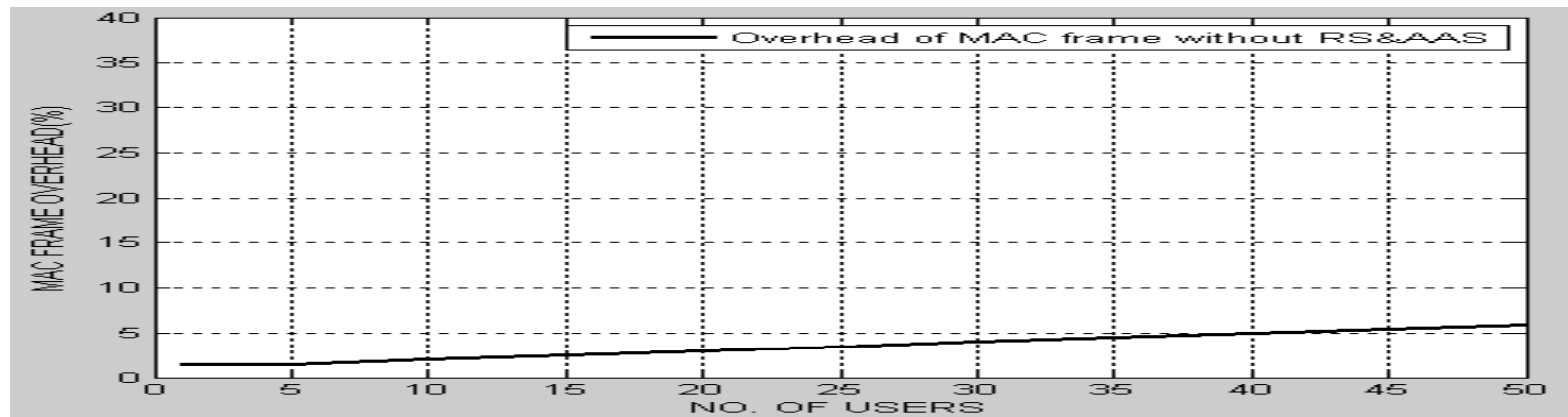

Figure (10) overhead of MAC frame without RS\&AAS. 
After including RSs and AAS to the original WiMAX cell, the percentage of overhead of MAC layer is demonstrated in Figure (11). It is obvious that the overhead of MAC layer will be increased by almost a factor of two due to the addition of RSs and AAS.

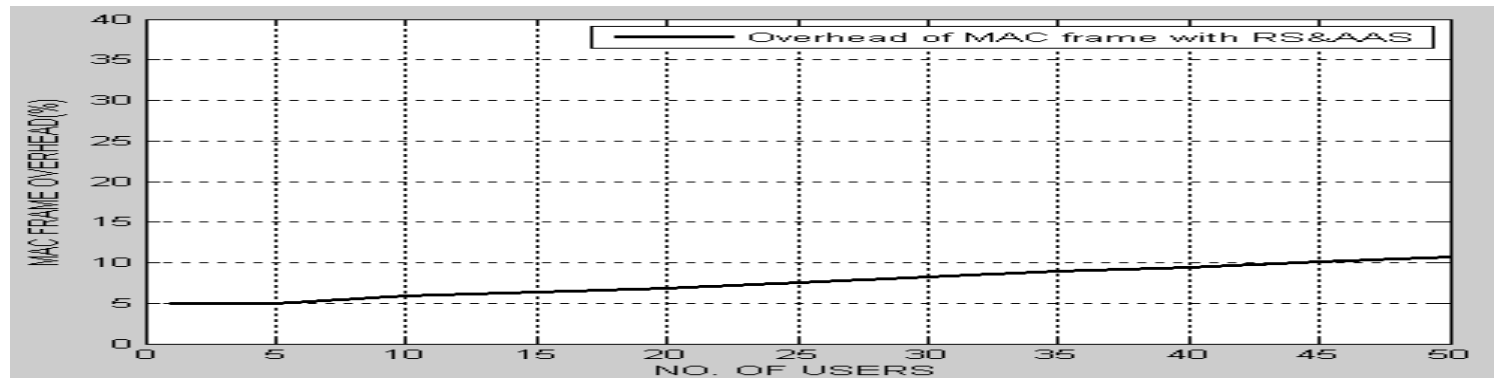

Figure(11) overhead of MAC frame with RS\&AAS.

Analytical results of $(\mathrm{Sc} / \mathrm{Rc})$ as a function of the number of users for the cases with and without RS and AAS are shown in Figure (12). For the purpose of comparison, the Figure also includes the OPNET results of Sc/Rc for the same cases. It is obvious that there is an excellent agreement between Analytical and OPNET results for all the values of the number of users equal or greater than (10). On the other hand, there is a slight difference between the two curves for the values of the number of users less than 10 which may be related to the modulation schemes available in OPNET (PSK modulated is not available).

In the case of without RS and AAS, the critical point on x-axis ( number of users) where the system capacity equal the requested capacity is equal to 17 users.

Any number of users less than (17) means that the system can provide the users with the services required, while the system fails to provide users with required services for all values greater than 17 (i.e. congestion occurs).

On the other hand, and in case of introducing RS and AAS to the cell (i.e. with RS and AAS), the critical point is equal to 22. This means that with RS and AAS there is an additional of about $30 \%$ of users that can utilize the system services without congestion.

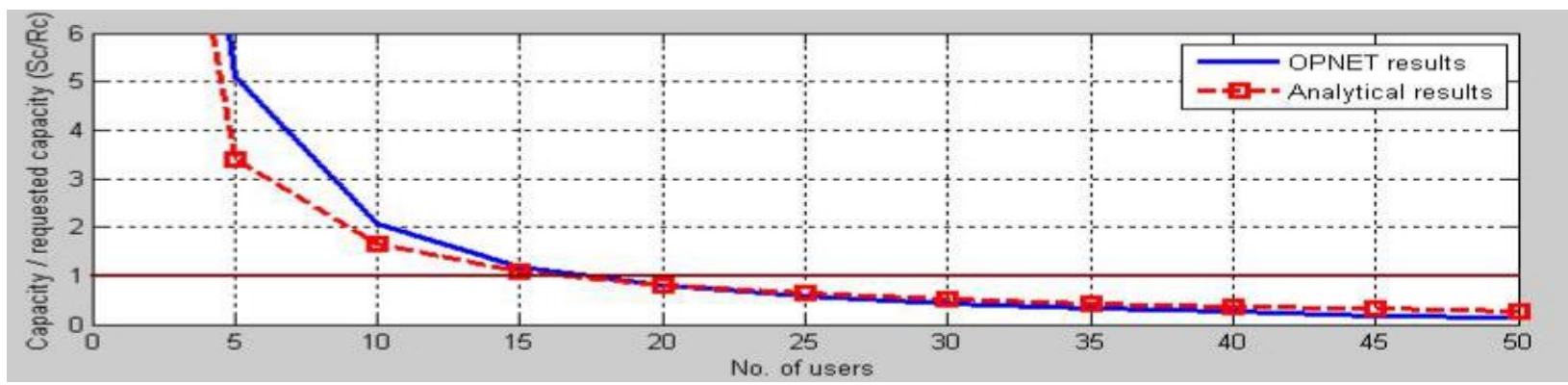

a-without RS and AAS

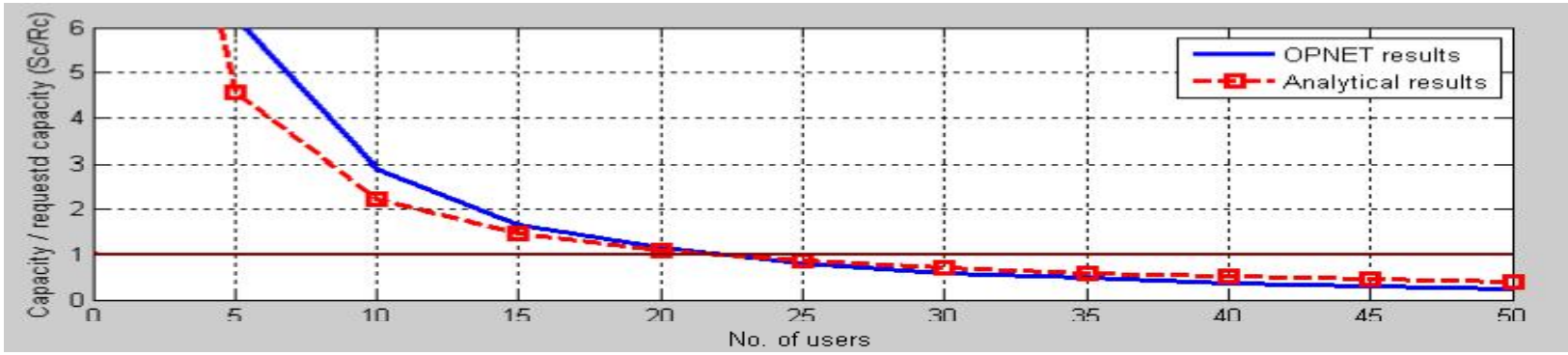

b- with RS and AAS.

Figure (12) Sc/Rc versus No. of users . 
Figure (13) illustrates the relationship between $\mathrm{Sc} / \mathrm{Rc}$ and the number of users for the cases with and without RS and AAS using analytical equations and for $B R=1 \mathrm{Mbps}$ and $\mathrm{BR}=4 \mathrm{Mbps}$.

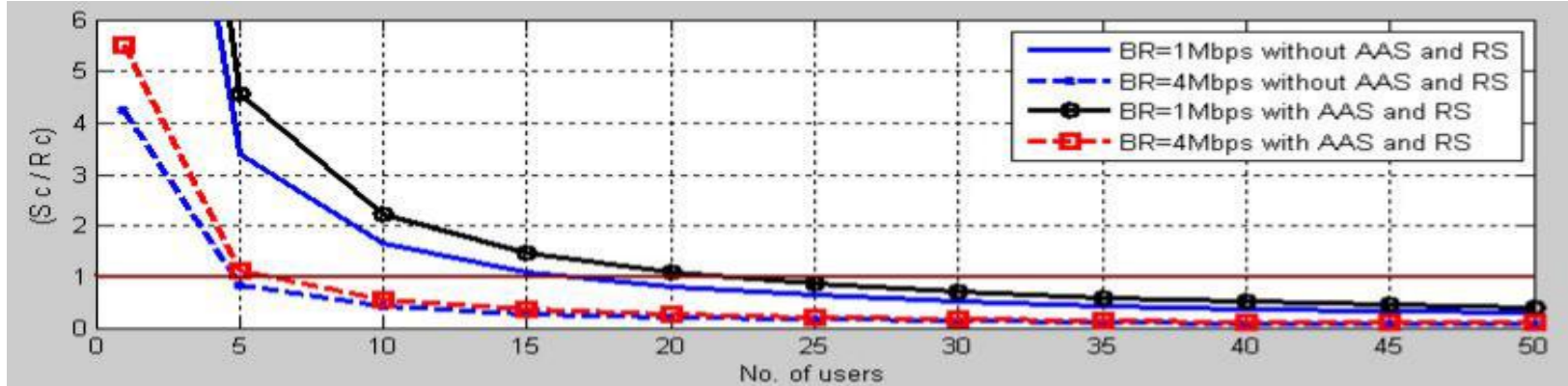

Figure (13) Sc/Rc versus the number of users for bit rates 1 and 4 Mbps respectively .

The Figure reveals that the number of users which can be served by the system decreases as the bit rate increases, this is related to the additional increment in the requested capacity. It is noted from the Figure that the system which is supported by AAS and RS can severe more users in comparison with the system without AAS and RS (for example at BR=4Mbps, the system without AAS and RS serve 4 users only while the system which is supported by AAS and RS can serve 6 users before congestion occurs ).

Figure (14) shows the relationship between $\mathrm{Sc} / \mathrm{Rc}$ and the nominal bit rate for given values of the number of users.

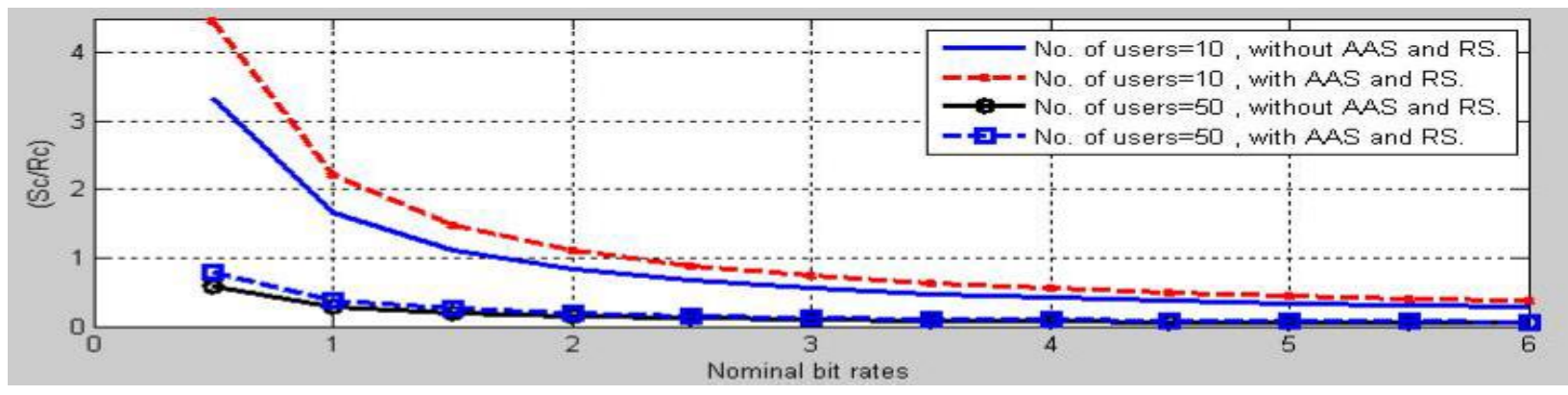

Figure (14) Shows Sc/Rc versus nominal bit rates.

Again the number of users to be served is decreased as the number of users increased, this is related to the increment of requested capacity.

Figure (15) illustrates the relationship between $\mathrm{Sc} / \mathrm{Rc}$ and the length of frames with and without RS and AAS and for two values of number of users (10 and 50) .

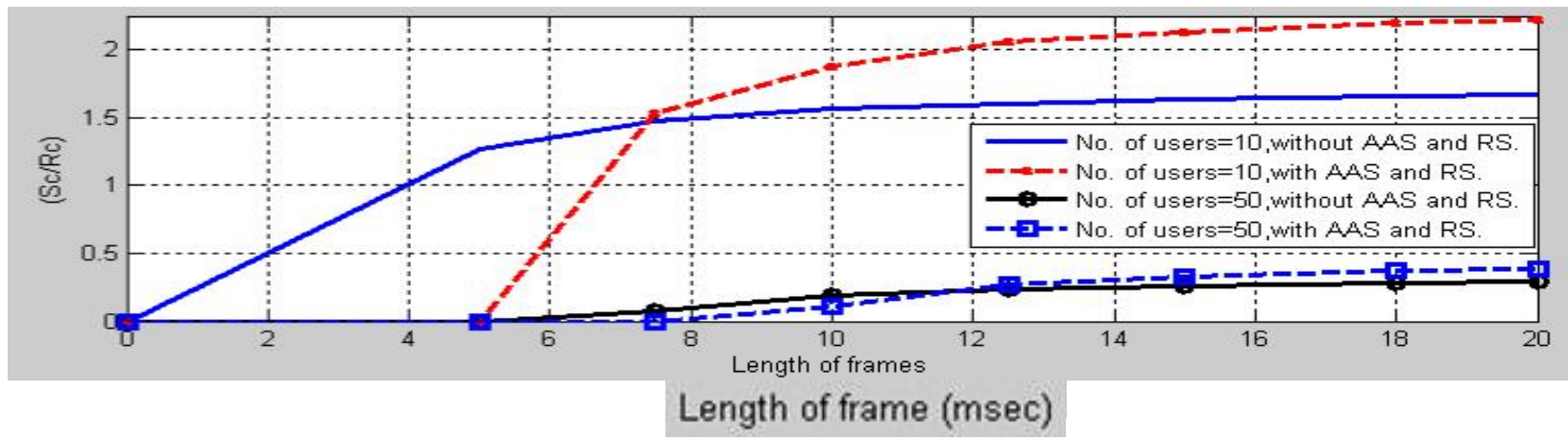

Figure (15) Shows Sc/Rc versus length of frames for two values of number of users. 
The Figures illustrate that as the number of users increase, the LOF (Length of frames) must be increased too to compensate for the additional overheads introduced by the users. It is also shown that the system which is supported by AAS and RS need more LOF than the system without AAS and RS to include the additional overheads that introduced by AAS and RS .

The Figure shows that for the ten users case and for all values of LOF equal or greater than $(7.5 \mathrm{msec})$ the advantage of introducing high level schemes of modulation (QPSK and 16-QAM) becomes apparent. This advantage is tumbled for the 50 users case which is due to the large amount of overheads introduced by the users, it is obvious very large LOF is needed to get the required benefit of the AAS and RS.

Throughput per user is shown in Figure (16) with and without AAS and RS . The curves show that at a number of users equal to 15 , there is an advantage of 1Mbps in the throughput performance with RS and AAS as compared with the throughput without RS and AAS .

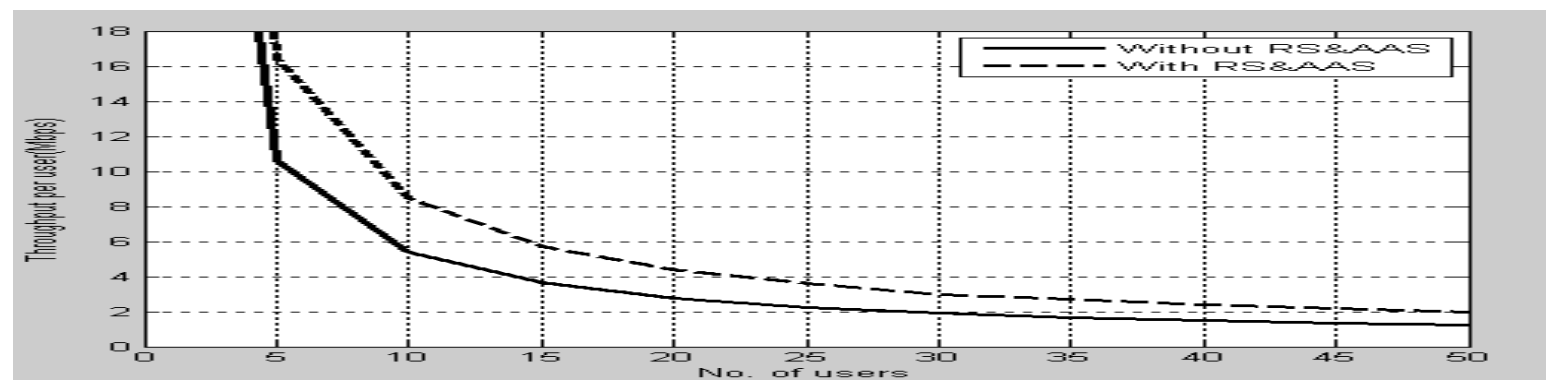

Figure (16) Throughput per user with and without AAS and RS .

\section{5-Conclusion}

This paper as mentioned before deals with the analysis and calculation of the overheads of PHY and MAC layers with and without Adaptive Antenna System (AAS) and relay stations (RS), it focusing on the effect of the number of users, nominal bit rates and length of frames on the system capacity to requested capacity and the throughput per user.The results show that the portion of the frame which is occupied by the overheads is significant, therefore and in the case of 50 users for example small length frames are not recommended ( LOF $<12 \mathrm{~ms}$ ) due to the small portion which is left for data transmission . The performance of system capacity is improved by employing the AAS and RS where at nominal bit rate equal to $1 \mathrm{Mbps}, 22$ users can utilize the system resources against 17 users for the system without RSs and AAS, before congestion occurs. The results show that the system capacity is deteriorated as the number of users and nominal bit rates are increased, which can be related to the increment of the requested capacity. It is worth to mention that some of the results are validated using OPNET version (14), excellent agreement between analytical and OPNET results are obtained especially when the number of users is equal or greater than (10).

\section{References}

[1] IEEE Std 802.16-2004, IEEE Standard for Local and metropolitan area networks , Part 16: air Interface for Fixed Broadband Wireless Access Systems, Oct. 04.

[2] Pavel Mach and Robert Bestak, "WiMAX performance evaluation", Department of 
Telecommunications Engineering Faculty of Electrical Engineering, Czech Technical University Prague, Czech Republic,2007.

[3] Pavel Mach and Robert Bestak, "Performance of IEEE 802.16 with relay stations", Department of Telecommunications Engineering, Czech Technical University, Technicka 2.

[4] Christian Hoymann, "Analysis and performance evaluation of the OFDM-based metropolitan area network IEEE802.16",Computer networks, 2005.

[5] Christian Hoymann ,Karsten Klagges and Marc Schinnenburg, "Multihop communication

6 , in relay inhanced IEEE 802.16 networks “, Chair of Communication Networks, Faculty

RWTH Aachen University,Germany, The 17th Annual IEEE International Symposium on

Personal,Indoor and Mobile Radio Communications (PIMRC'06).

[6] David Shani , "Smart antenna for wimax base station ", White Paper,VP of

International

Marketing \& Sales MTI Wireless Edge Ltd,2005.

[7] Y. Li and D Kenyon, "An examination of the processing complexity of an Adaptive Antenna System (AAS) for Wimax “,multiple access communication limited,UK, (C

IEE

2005 first presented at the $2{ }^{\text {nd }} I E E / E U R A S I P$ DSP enabled radio conference, September 2005 .

[8] Pavel Mach and Robert Bestak, "WiMAX Throughput Evaluation of Conventional Relaying “, springer, (C2008 .

[9] Aymen Belghith and Loutfi Nuaymi, "WiMAX capacity estimations and simulation results “, TELECOM and ENST Bretagne, France, 2 rue de la ch^ataigneraie, CS 17607 , 35576 .

[10] Sri Kiran Rangineni, "Multihop Concept in Cellular Systems “ , Master of Science Thesis

Gävle, Sweden 2008 . 